\title{
Renormalization of correlations in a quasiperiodically forced two-level system: quadratic irrationals
}

\author{
B. D. Mestel \\ Department of Computing Science and Mathematics \\ University of Stirling \\ Stirling \\ FK9 4LA, UK \\ B.D.Mestel@stir.ac.uk \\ A. H. Osbaldestin \\ Department of Mathematics \\ University of Portsmouth \\ Portsmouth \\ PO1 3HE, UK \\ andrew.osbaldestin@port.ac.uk
}

July 6,2004

\begin{abstract}
Generalizing from the case of golden mean frequency to a wider class of quadratic irrationals, we extend our renormalization analysis of the self-similarity of correlation functions in a quasiperiodically forced two-level system. We give a description of all piecewise-constant periodic orbits of an additive functional recurrence generalizing that present in the golden mean case. We establish a criterion for periodic orbits to be globally bounded, and also calculate the asymptotic height of the main peaks in the correlation function.
\end{abstract}




\section{Introduction}

Singular continuous spectra - indicative of a type of weak-mixing dynamics - have been observed by several authors for quasiperiodically forced two-level quantum systems in the case of piecewise-constant discontinuous modulation ([2], [5], [10]). Moreover, for the modulation frequencies studied, the autocorrelation function is found to possess asymptotic self-similar structure. In order to understand this self-similarity, Feudel et al [5] considered renormalization of the correlation function, found periodic orbits of the renormalization equations, and obtained the limiting behaviour of the principal peaks in the correlation function. Subsequently, in [13], we gave a mathematical analysis which justified and extended the numerical results in [5]. That analysis was restricted to the case of golden-mean frequency, which, because of its number-theoretic simplicity and dynamical robustness, has been often studied in quasiperiodic dynamical problems.

In this article we extend our work in [13] to consider the case of frequency equal to the quadratic irrational $\omega=\left(\sqrt{a^{2}+4}-a\right) / 2, a \in \mathbb{N}$, i.e., the irrational number with continued fraction expansion $[a, a, a, \ldots]$. This follows on from the work in [3], in which the strong-coupling fixed point for a renormalization for the Harper equation has been tackled.

The key to the analysis is the functional recurrence

$$
Z_{n}(x)=\left(\sum_{j=0}^{a-1} Z_{n-1}(-\omega x-j)\right)+Z_{n-2}\left(\omega^{2} x+a \omega\right),
$$

as seen in [3] in multiplicative form. The dynamics of this recurrence involve an interesting interplay between space and time evolution, with the behaviour on the whole real line being governed by a chaotic piecewise linear map $F$ (defined below) on a finite interval $I$, the fixed point set of an associated iterated function system on $\mathbb{R}$.

In this article we investigate the periodic orbits of the recurrence (1.1) for the case of piecewise constant functions $Z_{n}$. These orbits determine the self-similarity properties of the autocorrelation function for the discontinuous modulation considered in [5].

In our analysis of the periodic orbits of (1.1), we first of all consider the evolution of the discontinuity locations, and we show that the discontinuities must consist of finite unions of periodic orbits of $F$. A further constraint on the initial conditions $Z_{0}$ and $Z_{1}$ gives a necessary and sufficient condition for periodicity. In addition, we give an explicit criterion for the resulting periodic orbit to be spatially bounded on the real line.

Our work has strong connections with other problems involving the analysis of correlations in quasiperiodically driven systems, for example, the case of symmetric billiards in a square with a partial barrier ([1]), and the correlations in the fluctuations in a (generalized) Harper equation model of electron hopping $([3,14])$.

In the remainder of this introduction we review the quantum mechanical problem that motivates our work, the number-theoretic results that we shall draw on, and then the renormalization analysis of the autocorrelation function which gives the recurrence (1.1). In Section 2, we analyse the piecewise-constant periodic orbits of (1.1), and introduce the iterated function system and the chaotic map $F$ and the fundamental interval $I$ (referred to above). We analyse the dynamics of the discontinuity locations in terms of the dynamics of $F$, and, in particular, in terms of the periodic symbol sequences, or codes, which classify the periodic orbits of $F$. Finally, we determine conditions on the initial conditions $Z_{0}$ and $Z_{1}$ which, together with periodicity of the discontinuities, give periodic orbits $Z_{n}$ of (1.1). In Section 3, we extend the analysis of the discontinuities from the interval $I$ to the whole of $\mathbb{R}$ and determine the locations in $\mathbb{R}$ of the discontinuities generated by a discontinuity on $I$. In Section 4, we study spatial boundedness of periodic orbits, and, in Section 5, the preceding theory is harnessed to study in detail the periodic orbit generated by the modulation function 
considered in [5], and the limiting self-similar behaviour of autocorrelation function is calculated explicitly in this case. Finally, we give some directions for future development of this theory in our concluding remarks in Section 6.

\section{$1.1 \quad$ Equations of motion}

We briefly recall the quantum mechanical background to our problem. We refer the reader to [13] and the references therein for further details.

Let

$$
\sigma_{x}=\left(\begin{array}{cc}
0 & 1 \\
1 & 0
\end{array}\right), \quad \sigma_{y}=\left(\begin{array}{cc}
0 & -i \\
i & 0
\end{array}\right), \quad \sigma_{z}=\left(\begin{array}{rr}
1 & 0 \\
0 & -1
\end{array}\right) .
$$

denote the Pauli spin matrices. The Hamiltonian of a two-level system in a time-dependent magnetic field $B(t)$ can be wriiten $H=B(t) \cdot \sigma$ where $\sigma=\left(\sigma_{x}, \sigma_{y}, \sigma_{z}\right)$. For the special case $B(t)=(S(t) / 2,0, k / 2)$, the Hamiltonian is $H(t)=(k / 2) \sigma_{z}+(S(t) / 2) \sigma_{x}$. Taking $\hbar=1$, and writing the Schrödinger equation for a spinor $\psi=\left(\psi_{1}, \psi_{2}\right)$ in terms of the Bloch variables, i.e., the components of the polarization vector $P=\left(P_{x}, P_{y}, P_{z}\right)=\psi^{*} \sigma \psi$, we obtain the first order non-autonomous system of linear differential equations

$$
\dot{P}_{x}=-k P_{y}, \quad \dot{P}_{y}=k P_{x}-S(t) P_{z}, \quad \dot{P}_{z}=S(t) P_{y},
$$

subject to the normalization constraint $P_{x}^{2}+P_{y}^{2}+P_{z}^{2}=1$. For the case of forcing $S(t)$ consisting of periodic variable amplitude impulses with period $T$, we have $S(t)=\sum R_{n} \delta(t-n T)$, where $R_{n}$ is the amplitude at time $n$ and $\delta$ is the Dirac function.

Between impulses we have a rotation in the $\left(P_{x}, P_{y}\right)$ plane, and at impulses a rotation in the $\left(P_{y}, P_{z}\right)$ plane resulting in the linear impulse-to-impulse map

$$
\left(\begin{array}{c}
P_{x, n+1} \\
P_{y, n+1} \\
P_{z, n+1}
\end{array}\right)=\left(\begin{array}{rrr}
\cos k T & -\sin k T \cos R_{n} & \sin k T \sin R_{n} \\
\sin k T & \cos k T \cos R_{n} & -\cos k T \sin R_{n} \\
0 & \sin R_{n} & \cos R_{n}
\end{array}\right)\left(\begin{array}{c}
P_{x, n} \\
P_{y, n} \\
P_{z, n}
\end{array}\right)
$$

where we have written $\left(P_{x, n}, P_{y, n}, P_{z, n}\right)$ to denote the polarization vector at time step $n$.

Although the impulses occur periodically they do so with variable amplitude $R_{n}$ which we now take to be determined in a quasiperiodic manner governed by the rotation $\phi_{n+1}=\phi_{n}+\omega(\bmod 1)$ with the rotation number $\omega \notin \mathbb{Q}$. The amplitude $R_{n}$ is then defined via a period-1 modulation function $\Phi$ to be $R_{n}=\kappa \Phi\left(\phi_{n}\right)$ where $\kappa$ is an amplitude. The precise form of the modulation function $\Phi$ is crucial for the resulting dynamics. It is the case of piecewise-constant discontinuous modulation function, where a singular continuous spectrum is observed, that will concern us in this paper.

To simplify matters we assume that the time between impulses, $T$, is commensurate with the fundamental frequency $k$, setting $k T=2 \pi m, \in \mathbb{Z}$ thereby decoupling the variable $P_{x, n}$ so that the resulting dynamics is merely a rotation in the $\left(P_{y}, P_{z}\right)$ plane. (Numerical work in [5] indicates that this simplification may not be essential to witness the singular continuous spectrum.) Because of the constraint $P_{x}^{2}+P_{y}^{2}+P_{z}^{2}=1$, without loss of generality, we may set $P_{x, n}=0$ (otherwise we can simply rescale the remaining variables). Thus, writing $P_{y, n}=\cos \theta_{n}, P_{z, n}=\sin \theta_{n}$, we finally arrive at the skew-product system

$$
\phi_{n+1}=\phi_{n}+\omega(\bmod 1), \quad \theta_{n+1}=\theta_{n}+\kappa \Phi\left(\phi_{n}\right) .
$$

Of course it is straightforward to "solve" the system (1.5):

$$
\phi_{n}=\phi_{0}+n \omega \quad(\bmod 1), \quad \theta_{n}=\theta_{0}+\kappa \sum_{l=0}^{n-1} \Phi\left(\phi_{0}+l \omega\right),
$$


but this does not help illuminate the behaviour of the variable $\theta$, i.e., $\left(P_{y}, P_{z}\right)$. To this end we turn to an analysis of correlations, but before we do so we recall some elementary results on continued fractions.

\subsection{Number theoretic preliminaries}

Fix $a \in \mathbb{N}$ and define $\omega$ by the continued fraction

$$
\omega=\frac{1}{a+\frac{1}{a+\frac{1}{a+\cdots}}}=[a, a, \ldots],
$$

so that $\omega=\left(\sqrt{a^{2}+4}-a\right) / 2$. Note that $\omega$ satisfies the relations $\omega^{2}+a \omega=1$ and $\omega^{-1}=a+\omega$, which we shall use liberally in what follows. The rational convergents $p_{n} / q_{n}$ to $\omega$ are given by

$$
p_{n}=a p_{n-1}+p_{n-2}, \quad q_{n}=a q_{n-1}+q_{n-2},
$$

for $n \geq 1$, where we set

$$
p_{-1}=1, p_{0}=0 ; \quad q_{-1}=0, q_{0}=1 .
$$

Note that $p_{n}, q_{n}$ satisfy $p_{n}=q_{n-1}=G_{n}$ where

$$
G_{n}=a G_{n-1}+G_{n-2}, \quad G_{0}=0, \quad G_{1}=1,
$$

generalizing the Fibonacci numbers. An important relation which we shall often need is the identity

$$
G_{n-1} \omega=G_{n-2}-(-\omega)^{n-1},
$$

which may readily be proved by induction.

\subsection{Renormalization analysis of the autocorrelation function}

Let us recap briefly the renormalization analysis from [5] and [13], as adapted to the quadratic irrational $\omega$ for all $a \geq 1$. For a (zero mean) observable $P_{y}$, the autocorrelation function is

$$
K_{P_{y}}(t)=\frac{\left\langle P_{y, n} P_{y, n+t}\right\rangle}{\left\langle P_{y, n}^{2}\right\rangle}
$$

where we have using the notation

$$
\langle f(n)\rangle=\lim _{N \rightarrow \infty} \frac{1}{N} \sum_{n=1}^{N} f(n)
$$

for the time average of the observable $f(n)$ as $n \rightarrow \infty$. Now

$$
\left\langle P_{y, n} P_{y, n+t}\right\rangle=\left\langle\cos \theta_{n} \cos \theta_{n+t}\right\rangle=\frac{1}{2}\left\langle\cos \left(\theta_{n+t}-\theta_{n}\right)\right\rangle+\frac{1}{2}\left\langle\cos \left(\theta_{n+t}+\theta_{n}\right)\right\rangle=\frac{1}{2}\left\langle\cos \kappa Q_{t}\left(\phi_{n}\right)\right\rangle,
$$

where

$$
Q_{t}(\phi)=\sum_{l=0}^{t-1} \Phi(\phi+l \omega), \quad Q_{0}(\phi)=0,
$$

where the term $\cos \left(\theta_{n+t}+\theta_{n}\right)$ has been set to zero, by averaging over the initial phase $\theta_{0}$. Hence

$$
K_{P_{y}}(t)=\frac{\frac{1}{2}\left\langle\cos \kappa Q_{t}\left(\phi_{n}\right)\right\rangle}{\frac{1}{2}\left\langle\cos \kappa Q_{0}\left(\phi_{n}\right)\right\rangle}=\int_{0}^{1} \cos \kappa Q_{t}(\phi) d \phi,
$$


where, by ergodicity of the irrational rotation (1.5), we have replaced the time average by a space average. Now, at times $\left(G_{n}\right), Q_{n}$ satisfies the recurrence relation

$$
Q_{G_{n}}(\phi)=\sum_{j=0}^{a-1} Q_{G_{n-1}}\left(\phi+j G_{n-1} \omega\right)+Q_{G_{n-2}}\left(\phi+a G_{n-1} \omega\right) .
$$

Since the argument of $Q_{G_{n}}$ is defined modulo 1, we may use the identity (1.11) and define the rescaled variables

$$
Z_{n}(y)=Q_{G_{n}}\left(y(-\omega)^{n}\right),
$$

to obtain the iterative functional recurrence (1.1) with initial conditions

$$
Z_{0}(x)=0, \quad Z_{1}(x)=\Phi(-\omega x) .
$$

This recurrence is the object of study in this paper.

In terms of the functions $Z_{n}$, the autocorrelation function $K_{P_{y}}$ at times $G_{n}$, which we shall refer to as resonance times, is

$$
K_{P_{y}}\left(G_{n}\right)=\frac{1}{(-\omega)^{-n}} \int_{0}^{(-\omega)^{-n}} \cos \left(\kappa Z_{n}(y)\right) d y .
$$

For the particular choice of modulation function

$$
\Phi(\phi)= \begin{cases}+1 ; & 0 \leq\{\phi\}<1 / 2, \\ -1 ; & 1 / 2 \leq\{\phi\}<1,\end{cases}
$$

where $\{\phi\}$ denotes $\phi(\bmod 1)$, with the initial conditions given by (1.19), when $a=2$, iteration of (1.1) leads to the period-2 orbit as shown in Figure 1. In this case, the corresponding autocorrelation function $K_{P_{y}}$ (with $\kappa=\pi / 2$ ) is shown in Figure 2. Note that the largest peaks occur at every even resonance time, and that their height is approximately 0.65 , a value numerically calculated at even resonance times in [15] for the corresponding peak height in the strange nonchaotic attractor problem. By carefully describing the locations of the discontinuities in Figure 1, in Section 5 we shall explain why the peaks occur at even resonance times and moreover show that their asymptotic height is in fact $1-1 / \sqrt{8}=0.646446 \ldots$

In fact there is a dichotomy between even and odd $a$. For even $a$, a period- 2 orbit results, whilst for odd $a$ a period- 6 orbit results. When $a$ is even the peaks occur at every even resonance time, whilst for odd $a$ they occur at every third resonance time. In all cases their asymptotic height is $1-1 / \sqrt{a^{2}+4}$.

Our analysis shows that self-similarity of the autocorrelation function occurs for many other choices of modulation function in addition to (1.21). However for that to occur the locations of the discontinuities of the modulation function must be preperiodic points of a map of the interval to be introduced in the next section.

\section{Periodic orbits and their discontinuities}

We begin this section by adapting our previous analysis [12] of the multiplicative version of (1.1) to the problem at hand. We introduce an expanding piecewise-linear map of an interval whose periodic orbits correspond to the discontinuities of the piecewise-constant periodic orbits of (1.1), and show that the dynamics of this interval map 'drives' the global behaviour of periodic orbits of (1.1). We also give a detailed analysis of the periodicity of the discontinuities. We then give a necessary and sufficient criterion for the piecewise-constant orbits of (1.1) to be periodic. 

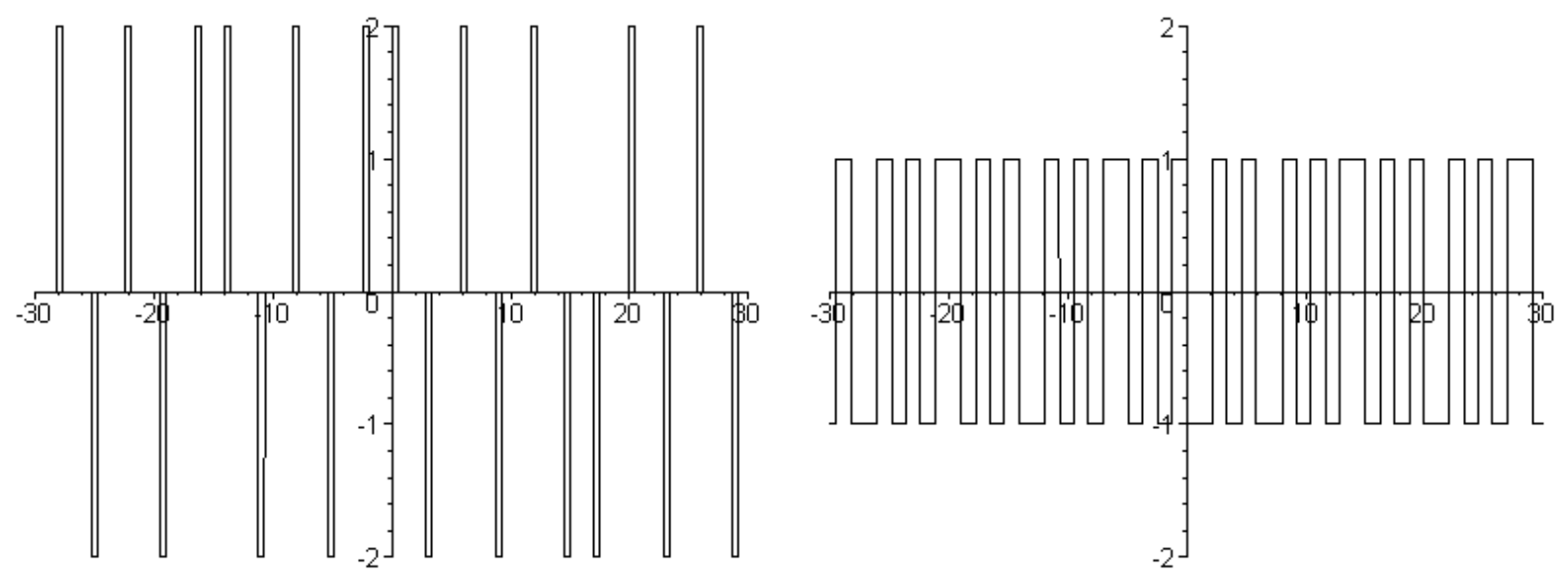

Figure 1: $Z_{n}(y)$ vs. $y$ for $n$ odd/even, showing the period-2 orbit resulting from iteration of (1.1) in the case $a=2$ with modulation function (1.21).

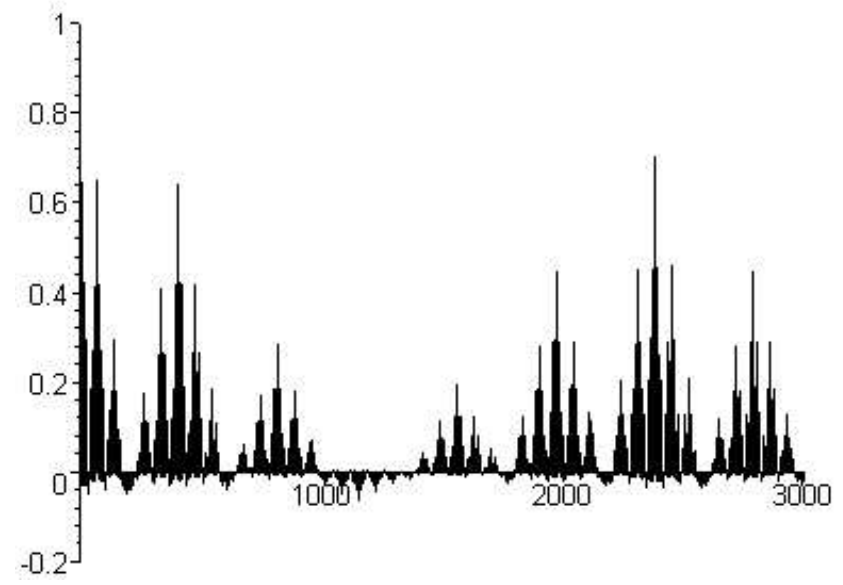

Figure 2: Autocorrelation function $K_{P_{y}}(t)$ vs. $t$ with $\kappa=\pi / 2$ in the case $a=2$ with modulation function (1.21).

\subsection{Iterated function system and the inverse map $F$}

Defining, for $j=0, \ldots, a-1$,

$$
\phi_{1, j}(x)=-\omega x-j, \quad \phi_{2, a}(x)=\omega^{2} x+a \omega,
$$

we may write equation (1.1) in the form

$$
Z_{n}(x)=\left(\sum_{j=0}^{a-1} Z_{n-1}\left(\phi_{1, j}(x)\right)\right)+Z_{n-2}\left(\phi_{2, a}(x)\right)
$$

The iterated function system (IFS) on $\mathbb{R}$ given by the linear contractions $\phi_{1,0}, \ldots, \phi_{1, a-1}, \phi_{2, a}$ has fundamental interval $I=[-\omega-a+1,1]$. Indeed $\phi_{1, j}(I)=[-\omega-j,-\omega-j+1], \phi_{2, a}(I)=[1-\omega, 1]$, so that $\bigcup_{j=0}^{a-1} \phi_{1, j}(I) \cup \phi_{2, a}(I)=I$. The fundamental interval $I$ is thus the attractor for the IFS.

Let $F: I \rightarrow I$ be defined by

$$
F(x)= \begin{cases}\phi_{1, j}^{-1}(x)=-(a+\omega) x-(a+\omega) j, \quad j=0, \ldots, a-1, & x \in[-\omega-j,-\omega-j+1) \\ \phi_{2, a}^{-1}(x)=\left(1+a^{2}+a \omega\right) x-a(a+\omega), & x \in[1-\omega, 1],\end{cases}
$$


as drawn in Figure 3 for the case $a=2$. We shall see below that periodic points of $F$ correspond to discontinuities of the periodic solutions of (2.2).

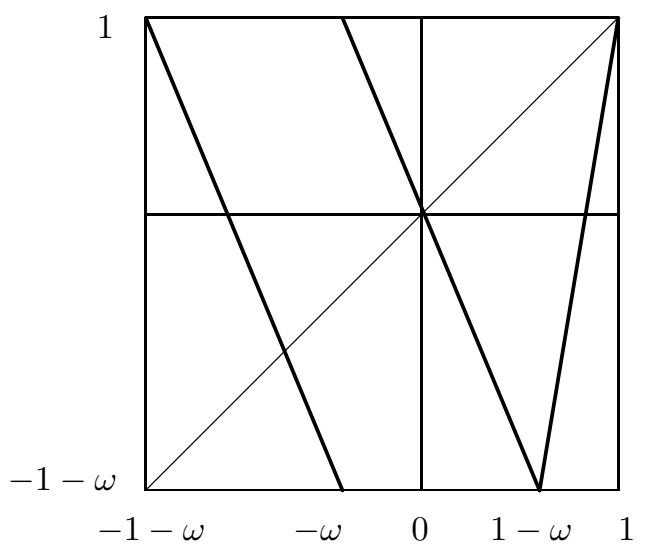

Figure 3: The function $F$ in the case $a=2$.

We note that for any periodic point $y \in I$, precisely one of $\phi_{1,0}(y), \ldots, \phi_{1, a-1}(y), \phi_{2, a}(y)$ is also a periodic point of $F$. This follows from the fact that each periodic point has $a+1$ preimages, exactly one of which is a periodic point on the same orbit.

We analyse the dynamics of $F$ in terms of a code of a point $x \in I$. We let the interval $(-\omega-j,-\omega-j+1)$ be encoded with the symbol $1^{j}$ and $(1-\omega, 1]$ with the symbol $2^{a}$. We define the code of $x \in I$ to be the sequence $\left(c_{n}^{b_{n}}\right)_{n \geq 0}$ in $\left\{1^{0}, \ldots, 1^{a-1}, 2^{a}\right\}^{\mathbb{N}_{0}}$ given by

$$
c_{n}^{b_{n}}= \begin{cases}1^{j}, & F^{n}(x) \in(-\omega-j,-\omega-j+1) ; \\ 2^{a}, & F^{n}(x) \in(1-\omega, 1],\end{cases}
$$

and ignore the (countable) set of (nonperiodic) points whose orbits under $F$ include the points $-\omega-l$, $l=-1,0, \ldots, a-1$. Periodic orbits of $F$ correspond to periodic codes in $\left\{1^{0}, \ldots, 1^{a-1}, 2^{a}\right\}^{\mathbb{N}_{0}}$ under the shift $\operatorname{map} \sigma$ :

$$
\sigma\left(c_{0}^{b_{0}} c_{1}^{b_{1}} c_{2}^{b_{2}} \ldots\right)=c_{1}^{b_{1}} c_{2}^{b_{2}} \ldots
$$

A periodic orbit $y_{0}, y_{1}, \ldots, y_{k-1}$ of period $k$ of $F$ is given uniquely by a periodic code $\overline{c_{0}^{b_{0}} c_{1}^{b_{1}} \ldots c_{k-1}^{b_{k-1}}}$, which we henceforth denote as just $c_{0}^{b_{0}} c_{1}^{b_{1}} \ldots c_{k-1}^{b_{k-1}}$. Indeed, given a code $c_{0}^{b_{0}} c_{1}^{b_{1}} \ldots c_{k-1}^{b_{k-1}}$, it is straightforward to calculate the corresponding periodic orbit $y_{0}, y_{1}, \ldots, y_{k-1}$ of $F$. We have $\phi_{c_{k-1}, b_{k-1}}^{-1} \circ \cdots \circ \phi_{c_{0}, b_{0}}^{-1}\left(y_{0}\right)=y_{0}$, or, equivalently, $\phi_{c_{0}, b_{0}} \circ \cdots \circ \phi_{c_{k-1}, b_{k-1}}\left(y_{0}\right)=y_{0}$, whose (unique) solution is readily calculated to be

$$
y_{0}=\frac{-\sum_{j=0}^{k-1} b_{j}(-\omega)^{-1+\sum_{i=0}^{j} c_{i}}}{1-(-\omega)^{\Sigma c_{j}}} .
$$

Indeed, noting that for $c^{b} \in\left\{1^{0}, \ldots, 1^{a-1}, 2^{a}\right\}$ we have $\phi_{c, b}=(-\omega)^{c} x-b(-\omega)^{-1+c}$. The formula $\phi_{c_{0}, b_{0}} \circ$ $\cdots \circ \phi_{c_{k-1}, b_{k-1}}\left(y_{0}\right)=(-\omega)^{\sum_{j=0}^{k-1} c_{j}} y_{0}-\sum_{j=0}^{k-1} b_{j}(-\omega)^{-1+\sum_{i=0}^{j} c_{i}}$ follows by induction, whence (2.6).

The other points of the orbit may be calculated by applying this formula with the code $c_{0}^{b_{0}} c_{1}^{b_{1}} \ldots c_{k-1}^{b_{k-1}}$ cyclically permuted.

For example, $F(y)$ has $a+1$ fixed points: $y=-j(a-1+\omega) / a$ with codes $1^{j}$, respectively, and $y=1$ with code $2^{a}$. The point $y_{0}=1 / 2$ is part of the period-two orbit $\{1 / 2,-\omega / 2\}$ with code $2^{1} 1^{0}$ when $a=1$, part of the period-three orbit $\{1 / 2,-(a+\omega) / 2,-(a-1) / 2-\omega / 2\}$ with code $1^{0} 1^{(a+1) / 2} 1^{(a-1) / 2}$ when $a$ is odd greater than one, and part of the period-two orbit $\{1 / 2,-(a+\omega) / 2\}$ with code $1^{0} 1^{a / 2}$ when $a$ is even.

It is the fixed point $y=0$ and the periodic orbit associated with $1 / 2$ that are the discontinuity points in the fundamental interval for the periodic orbit shown in Figure 1. 


\subsection{Reduction of $Z_{n}$ on $\mathbb{R}$ to the fundamental interval}

In this section we consider equation (2.2) outside the fundamental interval $I$, i.e., on the whole of $\mathbb{R}$. The fact that $I$ is the attractor for the IFS leads to the conclusion that the global behaviour of (2.2) is 'driven' by its behaviour in $I$.

The following result is a straightforward variation of the corresponding result in [12].

Lemma 1. Let $Z_{0}, Z_{1}$ be initial conditions for (2.2) on $\mathbb{R}$ and let $\varepsilon>0$ be such that $Z_{0}(x)=Z_{1}(x)=0$ for all $x \in[-\omega-(a-1)-\varepsilon, 1+\varepsilon]$. Then for each $L>1$, there exists $N>0$ (depending only on $L$ ) such that $Z_{n}(x)=0$ for all $x \in[-L, L]$ and all $n>N$.

In other words, if the initial conditions on, and just outside, the fundamental interval are zero, then the value of $Z_{n}$ at all points eventually becomes zero.

From the lemma we may prove the following proposition whose proof again follows mutatis mutandis the corresponding result in [12].

Proposition 1. Let $Z_{n}$ be a piecewise-constant periodic orbit of (2.2) of period $p$ on $\mathbb{R}$ with $Z_{n}(1+)=Z_{n}(1)$. Then $Z_{n}$ is periodic with period $p$ on the fundamental interval $I$. Conversely, suppose that $Z_{n}$ is periodic with period $p$ on $I$. Then there is a unique extension $\tilde{Z}_{n}$ of $Z_{n}$ to $\mathbb{R}$ such that $\tilde{Z}_{n}$ is periodic with period $p$ on $\mathbb{R}$.

Moreover Lemma 1 further implies the following.

Proposition 2. Let $Z_{0}, Z_{1}$ be piecewise-constant initial conditions for (2.2) on $\mathbb{R}$ with $Z_{0}(1+)=Z_{0}(1)$, $Z_{1}(1+)=Z_{1}(1)$. Suppose $Z_{n}$ is periodic of period $p$ on the fundamental interval $I$. Then the sequence $Z_{n}$ converges to the unique periodic extension $\tilde{Z}_{n}$ given by proposition 1, i.e., for all integers $r \geq 0$ we have $Z_{r+n p}(x) \rightarrow \tilde{Z}_{r}(x)$ as $n \rightarrow \infty$.

In other words, initial data on the fundamental interval resulting in periodic behaviour uniquely determines an asymptotic (right continuous at 1) global periodic behaviour. It is important to realise however that such globally defined periodic orbits will not in general consist of bounded functions. We shall address this issue in Section 4.

\subsection{Analysis of the discontinuities}

In order to study the piecewise-constant periodic orbits of the recurrence (1.1) we firstly consider the dynamics of the discontinuities. We define, for each $x \in \mathbb{R}$ and $n \geq 0$,

$$
D_{n}(x)=Z_{n}(x+)-Z_{n}(x-),
$$

the difference of the right-hand limit at $x$ to the left-hand limit at $x$, so that $D_{n}(x) \neq 0$ if and only if $Z_{n}$ has a discontinuity at $x$. The recurrence (2.2) for $Z_{n}$ naturally induces a recurrence for $D_{n}$ :

$$
\begin{aligned}
D_{n}(x) & =Z_{n}(x+)-Z_{n}(x-) \\
& =\sum_{j=0}^{a-1} Z_{n-1}\left(\phi_{1, j}(x+)\right)+Z_{n-2}\left(\phi_{2, a}(x+)\right)-\sum_{j=0}^{a-1} Z_{n-1}\left(\phi_{1, j}(x-)\right)-Z_{n-2}\left(\phi_{2, a}(x-)\right) \\
& =\sum_{j=0}^{a-1}\left(Z_{n-1}\left(\phi_{1, j}(x)-\right)-Z_{n-1}\left(\phi_{1, j}(x)+\right)\right)+Z_{n-2}\left(\phi_{2, a}(x)+\right)-Z_{n-2}\left(\phi_{2, a}(x)-\right) \\
& =-\sum_{j=0}^{a-1} D_{n-1}\left(\phi_{1, j}(x)\right)+D_{n-2}\left(\phi_{2, a}(x)\right) .
\end{aligned}
$$


Clearly if $Z_{n}$ is periodic with period $p$, then $D_{n}$ is also periodic with period $m$ dividing $p$. Our task is to determine the possible periods $m$ of $D_{n}$ and relate $m$ to $p$, the period of $Z_{n}$.

From now on we assume that $Z_{n}$ is periodic with period $p$ and that $D_{n}$ is periodic with period $m$, and, in view of Proposition 1, we only consider the behaviour of $Z_{n}$ and $D_{n}$ on the fundamental interval $I$. We define the restricted discontinuity set

$$
D=\left\{x \in I: D_{n}(x) \neq 0 \text { for some } n \geq 0\right\} .
$$

Then $D$ is the finite set of points in $I$ for which $Z_{n}$ has a discontinuity for at least one $n \geq 0$.

As in our analysis of the multiplicative recurrence [12], the restricted discontinuity set $D$ consists of finitely many periodic orbits of the map $F$ as we now show.

Proposition 3. Let $Z_{n}$ be a piecewise-constant periodic orbit of (2.2), and let $D$ be the associated restricted discontinuity set. Then $D$ consists of a finite collection of periodic orbits of the map $F$.

Proof. Suppose $y \in D$. Then $D_{n}(y) \neq 0$ for some $n \geq 0$. From (2.8) we have that $D_{n-i_{1}}\left(\phi_{i_{1}, j_{1}}(y)\right) \neq 0$ for some $i_{1} \in\{1,2\}, j_{1} \in\{0, \ldots, a\}$. . We therefore have $\phi_{i_{1}, j_{1}}(y) \in D$. Continuing in this way, we obtain a sequence of pairs $\left(i_{1}, j_{1}\right),\left(i_{2}, j_{2}\right), \ldots$ such that $\phi_{i_{k}, j_{k}} \circ \cdots \circ \phi_{i_{1}, j_{1}}(y) \in D$ for each $k \in \mathbb{N}$. Since $D$ is finite there exist $r, r^{\prime} \in \mathbb{N}$ with $r>r^{\prime}$ and $\phi_{i_{r^{\prime}}, j_{r^{\prime}}} \circ \cdots \circ \phi_{i_{1}, j_{1}}(y)=\phi_{i_{r}, j_{r}} \circ \cdots \circ \phi_{i_{1}, j_{1}}(y)$. Applying $F^{r}$ to this equation gives $F^{r-r^{\prime}}(y)=y$, so that $y$ is a periodic point of $F$ of period $j$ dividing $r-r^{\prime}$.

Now let $y_{0}=y, y_{1}, \ldots, y_{k-1}$ be the points on the orbit of $y_{0}$ under $F$ with $y_{i+1}(\bmod k)=F\left(y_{i}\right)$ for $i=$ $0,1, \ldots, k-1$, and let $c_{0}^{b_{0}} c_{1}^{b_{1}} \ldots c_{k-1}^{b_{k-1}}$ be the code of the orbit. Then for $0 \leq i \leq k-1$ we have

$$
\phi_{c_{i}, b_{i}}^{-1}\left(y_{i}\right)=y_{i+1}, \quad \text { or, equivalently, } \quad \phi_{c_{i-1}, b_{i-1}}\left(y_{i}\right)=y_{i-1},
$$

where here, and in what follows, we assume that indices relating to the periodic orbit $y_{0}, y_{1}, \ldots, y_{k-1}$ are reduced modulo $k$.

Moreover, as we noted earlier, precisely one of $\phi_{1,0}\left(y_{i}\right), \ldots \phi_{1, a-1}\left(y_{i}\right), \phi_{2, a}\left(y_{i}\right)$ is periodic. Thus, if $c_{i-1}=$ 1 then $\phi_{2, a}\left(y_{i}\right) \notin D$ and so $D_{n}\left(\phi_{2, a}\left(y_{i}\right)\right)=0$ for all $n$, whilst if $c_{i-1}=2$ then $\phi_{1, j}\left(y_{i}\right) \notin D$ and so $D_{n-1}\left(\phi_{1, j}\left(y_{i}\right)\right)=0$ for all $n$. The recurrence (2.8) may be therefore be written

$$
D_{n}\left(y_{i}\right)= \begin{cases}-D_{n-1}\left(y_{i-1}\right), & c_{i-1}=1 ; \\ D_{n-2}\left(y_{i-1}\right), & c_{i-1}=2,\end{cases}
$$

which can be written as

$$
D_{n}\left(y_{i}\right)=(-1)^{c_{i-1}} D_{n-c_{i-1}}\left(y_{i-1}\right) .
$$

Thus $D_{n+c_{0}+\cdots+c_{i-1}}\left(y_{i}\right)=(-1)^{c_{0}+\cdots+c_{i-1}} D_{n}\left(y_{0}\right)$, so that if $D_{n}\left(y_{0}\right) \neq 0$ then $D_{n+c_{0}+\cdots+c_{i-1}}\left(y_{i}\right) \neq 0$, i.e., if $y_{0} \in D$ then $y_{i} \in D$. We conclude that not only must every point $y \in D$ be periodic point of $F$, but that every point on the periodic orbit of $y$ also lies in $D$, so that $D$ consists of complete orbits of $F$.

\subsection{Period of the discontinuities for a single periodic orbit of $F$}

From (2.12) we see that over a complete periodic orbit with code $c_{0}^{b_{0}} c_{1}^{b_{1}} \ldots c_{k-1}^{b_{k-1}}$ the index $n$ decreases by

$$
l=\sum_{i=0}^{k-1} c_{i},
$$

and moreover we have $D_{n}\left(y_{i}\right)=(-1)^{l} D_{n-l}\left(y_{i}\right)$, for $0 \leq i \leq k-1$. We therefore deduce the following result. 
Proposition 4. Let $m$ be the period of the discontinuity function $D_{n}$ restricted to a periodic orbit $y_{0}, \ldots, y_{k-1}$ of $F$ and let $l$ be the sum of the code over the orbit of $F$. Then $m$ divides $l c m\{2, l\}$, i.e., if $l$ is even $m$ divides $l$, whilst if $l$ is odd $m$ divides $2 l$.

We define for any integer $m$

$$
\hat{m}=\operatorname{lcm}\{2, m\}=\left\{\begin{array}{lll}
m, & m & \text { even } ; \\
2 m, & m & \text { odd } .
\end{array}\right.
$$

A convenient representation of the discontinuity data is in terms of an $\hat{l} \times k$ matrix $M$, the discontinuity matrix, with entries defined by

$$
M_{n, i}=D_{n}\left(y_{i}\right),
$$

for $0 \leq n \leq \hat{l}-1,0 \leq i \leq k-1$, so that the entry in row $n$ and column $i$ is the value of $D_{n}$ at the point $y_{i}$ on the orbit $y_{0}, y_{1}, \ldots, y_{k-1}$.

The relation (2.12) above gives a special structure to the matrix $M$. Indeed (2.12) translates to

$$
M_{n, i}=(-1)^{c_{i-1}} M_{n-c_{i-1}, i-1},
$$

where here, and in what follows, indices referring to the periodicity of $D_{n}$ are reduced modulo $\hat{l}$.

The structure (2.16) can be more easily understood as follows. Column $i$ of the matrix $M$ is simply column $(i-1)$ cyclically permuted downwards by $c_{i-1}$ single cyclic permutations with a change of sign if $c_{i-1}=1$. This observation also holds when $i=0$, for then (2.16) becomes

$$
M_{n, 0}=(-1)^{c_{k-1}} M_{n-c_{k-1}, k-1} .
$$

Let us denote the first column of $M$ by $\left(X_{0}, X_{1}, \ldots, X_{\hat{l}-1}\right)$, i.e., $M_{n, 0}=X_{n}$ for $0 \leq n \leq \hat{l}-1$. Then the relation (2.16) tells us that

$$
M_{n, 1}=(-1)^{c_{0}} M_{n-c_{0}, 0}=(-1)^{c_{0}} X_{n-c_{0}}
$$

and, in general,

$$
M_{n, i}=(-1)^{\sum_{j=0}^{i-1} c_{j}} M_{n-\sum_{j=0}^{i-1} c_{j}, 0}=(-1)^{\sum_{j=0}^{i-1} c_{j}} X_{n-\sum_{j=0}^{i-1} c_{j}},
$$

so that each column of $M$ is simply a cyclic permutation with an appropriate sign change of the first column of $M$. In the case of odd $l$, in order to satisfy (2.17), the first column of $M$ must take the form $\left(X_{0}, X_{1}, \ldots, X_{l-1},-X_{0},-X_{1}, \ldots,-X_{l-1}\right)$.

Example 1. The periodic orbit generated by $1 / 2$.

When $a=1$, the period-two orbit $\{1 / 2,-\omega / 2\}$ has code $2^{1} 1^{0}$, so $k=2, l=3, \hat{l}=6$ and

$$
M=\left(\begin{array}{rr}
X_{0} & -X_{1} \\
X_{1} & -X_{2} \\
X_{2} & X_{0} \\
-X_{0} & X_{1} \\
-X_{1} & X_{2} \\
-X_{2} & -X_{0}
\end{array}\right),
$$

in which, since $c_{0}=2$, the second column is the first column shifted down two rows without sign change. Utilising $c_{1}=1$ on the second column reproduces the first column by shifting down one and changing sign. 
When $a$ is odd greater than one, the period-three orbit $\{1 / 2,-(a+\omega) / 2,-(a-1+\omega) / 2\}$ has code $1^{0} 1^{(a+1) / 2} 1^{(a-1) / 2}$, so $k=3, l=3, \hat{l}=6$ and

$$
M=\left(\begin{array}{rrr}
X_{0} & X_{2} & -X_{1} \\
X_{1} & -X_{0} & -X_{2} \\
X_{2} & -X_{1} & X_{0} \\
-X_{0} & -X_{2} & X_{1} \\
-X_{1} & X_{0} & X_{2} \\
-X_{2} & X_{1} & -X_{0}
\end{array}\right) .
$$

When $a$ is even the period-two orbit $\{1 / 2,-(a+\omega) / 2\}$ has code $1^{0} 1^{a / 2}$ so $k=l=\hat{l}=2$ and

$$
M=\left(\begin{array}{ll}
X_{0} & -X_{1} \\
X_{1} & -X_{0}
\end{array}\right)
$$

Example 2. The fixed point 0 has code $1^{0}$ so here $k=l=1, \hat{l}=2$ and

$$
M=\left(\begin{array}{r}
X_{0} \\
-X_{0}
\end{array}\right) .
$$

The combination of Examples 1 and 2 is precisely that required to study correlations with the choice (1.21), and is the initial motivation for studying this problem. It enables us to give a precise description of the numerical results of Feudel et al [5] and related results in [15]. We shall return to this example later on this article.

Example 3. The fixed point 1 has code $2^{a}$ so here $k=1, l=\hat{l}=2$ and

$$
M=\left(\begin{array}{c}
X_{0} \\
X_{1}
\end{array}\right) .
$$

Example 4. When the code is $2^{a} 1^{j_{1}} 1^{j_{2}} 2^{a}$ we have $k=4, l=\hat{l}=6$ and

$$
M=\left(\begin{array}{cccc}
X_{0} & X_{4} & -X_{3} & X_{2} \\
X_{1} & X_{5} & -X_{4} & X_{3} \\
X_{2} & X_{0} & -X_{5} & X_{4} \\
X_{3} & X_{1} & -X_{0} & X_{5} \\
X_{4} & X_{2} & -X_{1} & X_{0} \\
X_{5} & X_{3} & -X_{2} & X_{1}
\end{array}\right) .
$$

When $l$ is even, by a suitable choice of first column, i.e., of the $l$ numbers $X_{0}, X_{1}, \ldots, X_{l-1}$, we may arrange for the discontinuities to have any period dividing $l$. When $l$ is odd, by suitably choosing $X_{0}, X_{1}, \ldots, X_{l-1}$, any period twice any divisor of $l$ can be obtained (or the trivial period- 1 case of no discontinuities). Moreover, in this latter case, the discontinuities satisfy $X_{n+l}=-X_{n}$.

We emphasise that our freedom of choice is in choosing the values of the discontinuities at each point of a periodic orbit of $F$. It is not the case that one may arbitrarily select the discontinuities of the initial conditions $Z_{0}, Z_{1}$. This would correspond to specifying the first two rows of the discontinuity matrix. As our analysis shows, however, the rows are not independent. However, we remark that each row of the discontinuity matrix contains each $X_{i}$ at most once, and that any two adjacent rows together contain each $X_{i}$ at least once. Indeed, given one row, it is clear that those entries which appear in the following row are simply those in the columns corresponding to $y_{i}$ with code 1 . Thus an alternative (equivalent) method of specifying 
a periodic discontinuity orbit may be given directly in terms of the discontinuities of $Z_{0}$ and $Z_{1}$ at each $y_{i}$. Namely, the discontinuities of $Z_{1}$, i.e., $D_{1}\left(y_{i}\right)$ may be chosen arbitrarily, as may the the discontinuities $D_{0}\left(y_{i}\right)$ of $Z_{0}$ at each $y_{i}$ with code 2 . (This exhausts $l$ freedoms.) The discontinuities of $Z_{0}$ at each $y_{i}$ with code 1 , however are given as $D_{0}\left(y_{i}\right)=-D_{1}\left(y_{i+1}\right)$ (with period $k$ on the index). Whilst useful for ensuring that initial conditions result in periodic behaviour, this alternative description is less useful for determining the precise period. This description will however be useful in Section 4 when we wish to analyse the global behaviour of recurrence $(2.2)$.

\subsection{Multiple periodic orbits in $D$}

In general, the restricted discontinuity set $D$ will be composed of points of more than one periodic orbit of $F$. Let $t$ be the number of periodic orbits of $F$ in $D$. For $0 \leq s \leq t-1$, we consider the periodic orbit $s$ of $F$ in $D$. We make the general convention that superscript $s$ refers to the orbit $s$. Now, from the additive structure of (2.8), we have that a sum of solutions is again a solution of the equation. Moreover, because the periodic orbits in $D$ are distinct, and are never mapped to each other under the two maps $\phi_{1, j}, \phi_{2, a}$, we have that the dynamics of $D_{n}$ on each of the periodic orbits in $D$ are independent. Indeed, we may write

$$
D_{n}(x)=\sum_{s=0}^{t-1} D_{n}^{s}(x)
$$

where $D_{n}^{s}$ is the restriction of $D_{n}$ to the periodic orbit $s$, i.e.,

$$
D_{n}^{s}(x)= \begin{cases}D_{n}(x), & x \in\left\{y_{0}^{s}, \ldots, y_{k^{s}-1}^{s}\right\} \\ 0, & \text { otherwise }\end{cases}
$$

We may then apply the analysis of the previous subsection to each of the functions $D_{n}^{s}$. This is because $D_{n}^{s}(x)=0$, except when $x$ is one of the points on the periodic orbit $y_{0}^{s} \ldots y_{k^{s}-1}^{s}$ of $F$.

The theory for $D_{n}$ that we discussed above carries over in a straightforward manner to the function $D_{n}^{s}$. In particular, for each orbit in $D$ we can formulate an $\hat{l}^{s} \times k^{s}$ discontinuity matrix $M^{s}$, defined by $M_{n, i}^{s}=D_{n}^{s}\left(y_{i}^{s}\right)$ for $0 \leq n \leq \hat{l}^{s}-1$ and $0 \leq i \leq k^{s}-1$. To simplify notation, we adopt the convention that matrix indices are reduced modulo $k^{s}$ when dealing with expressions relating to the periodic orbit $y_{0}^{s}, \ldots, y_{k^{s}-1}^{s}$ of $F$, whilst those relating to the periodicities of $D_{n}$ are reduced modulo $\hat{l}^{s}$. These matrices are independent of each other since the dynamics of $D_{n}$ on each periodic orbit in $D$ are independent.

Thus, as in (2.16), we have

$$
M_{n, i}^{s}=(-1)^{c_{i-1}^{s}} M_{n-c_{i-1}^{s}, i-1}^{s},
$$

for $0 \leq n \leq \hat{l}^{s}-1$ and $0 \leq i \leq k^{s}-1$, and the matrix $M^{s}$ is determined by its first column $\left(X_{0}^{s}, X_{1}^{s}, \ldots, X_{\hat{l}^{s}-1}^{s}\right)$. (When $l^{s}$ is odd this column takes the form $\left(X_{0}^{s}, X_{1}^{s}, \ldots, X_{l^{s}-1}^{s},-X_{0}^{s},-X_{1}^{s}, \ldots,-X_{l^{s}-1}^{s}\right)$.)

Indeed, as in (2.19),

$$
M_{n, i}^{s}=(-1)^{\sum_{j=0}^{i-1} c_{j}^{s}} X_{n-\sum_{j=0}^{i-1} c_{j}^{s}}^{s},
$$

and the period $m^{s}$ of the first column is precisely the row period of $M^{s}$. We also have $m^{s} \mid \hat{l}^{s}$.

Conversely, let $\hat{l}=\operatorname{lcm}\left(\hat{l}^{0}, \ldots, \hat{l}^{t-1}\right)$. Then for any $m \mid \hat{l}$ we define $m^{s}=\operatorname{gcd}(m, \hat{l})$. Then $m^{s} \mid \hat{l}^{s}$ and by appropriate choices of $\left(X_{0}^{s}, X_{1}^{s}, \ldots, X_{\hat{l}^{s}-1}^{s}\right)$ we may construct a matrix $M^{s}$ with row period $m^{s}$ if $l^{s}$ is even, or $\hat{m}^{s}$ if $l^{s}$ is odd, and, extending periodically to all $n \geq 0$, we have that $D_{n}$ has period $m^{s}$ if $l^{s}$ is even, or $\hat{m}^{s}$ if $l^{s}$ is odd, when restricted to the orbit $y_{0}^{s}, \ldots, y_{k^{s}-1}^{s}$.

We therefore have the following proposition. 
Proposition 5. Let $Z_{n}$ be a piecewise-constant periodic orbit of (2.2). Then the period $m$ of the discontinuity function $D_{n}$ is given by

$$
m=\operatorname{lcm}\left(m^{0}, \ldots, m^{t-1}\right),
$$

where $m^{s}$ is the period of the function $D_{n}^{s}$, given by the period of $\left(X_{0}^{s}, X_{1}^{s}, \ldots, X_{\hat{l}^{s}-1}^{s}\right)$, i.e., the first column of the discontinuity matrix $M^{s}$. Furthermore, $m$ divides

$$
\hat{l}=\operatorname{lcm}\left(\hat{l}^{0}, \ldots, \hat{l}^{t-1}\right) .
$$

Moreover, by appropriate choices of $\left(X_{0}^{s}, X_{1}^{s}, \ldots, X_{\hat{l}^{s}-1}^{s}\right)$, for any $m$ dividing $\hat{l}$ we may construct a periodic orbit of $D_{n}$ with period $\hat{m}$ (and, if all $l^{s}$ are even, we may construct a periodic orbit of odd period $m$ ).

\subsection{The criterion for orbits to be periodic}

In the previous subsection we dealt quite extensively with the periodicity of the discontinuities on the fundamental interval $I$. It is perfectly feasible for the sequence of discontinuities $D_{n}$ to be periodic whilst the sequence $\left(Z_{n}\right)$ itself is not. The simple example $Z_{0}(x)=0, Z_{1}(x)=1$ for all $x$, with no discontinuities at all, generates the sequence $\left(G_{n}\right)$ as the values for $Z_{n}$. This sequence is clearly not periodic, growing without bound.

In order to ensure that $\left(Z_{n}\right)$ is itself periodic it suffices to ensure that the values at a single point are periodic. It will be convenient to choose an end point of $I$ for this purpose. We consider then the values $Z_{n}(1)$. As we saw in Subsection 2.2, it is not just the fundamental interval, but also the right hand limit at the end point 1 of the interval that dictates the global behaviour. With this in mind we take $Z_{n}$ to be right continuous at 1 and write $Z_{n}^{1+}=Z_{n}(1)=Z_{n}(1+)$.

The recurrence $(2.2)$ gives

$$
Z_{n}(1+)=\sum_{j=0}^{a-1} Z_{n-1}\left(\phi_{1, j}(1+)\right)+Z_{n-2}\left(\phi_{2, a}(1+)\right) .
$$

Now $\phi_{2, a}$ is increasing and $\phi_{2, a}(1)=1$, so $\phi_{2, a}(1+)=1+$, and thus $Z_{n-2}\left(\phi_{2, a}(1+)\right)=Z_{n-2}(1+)$. On the other hand each $\phi_{1, j}$ is decreasing and $\phi_{1, j}(1)=-\omega-j$, so now $\phi_{1, j}(1+)=(-\omega-j)-$. To relate $Z_{n-1}\left(\phi_{1, j}(1+)\right)$ to $Z_{n-1}(1+)$ we therefore need to pass from $(-\omega-j)-$ to $1+$, which means that we need to add in the effect of all of the discontinuities of $Z_{n-1}$ in $[-\omega-j, 1]$. For each $j$, let us therefore write

$$
Z_{n-1}(1+)=Z_{n-1}(-\omega-j-)+\sigma_{n-1}^{j}
$$

where we denote

$$
\sigma_{n-1}^{j}=\sum_{y \in[-\omega-j, 1]} D_{n-1}(y) .
$$

We thus have the following recurrence relation for $Z_{n}^{1+}$ :

$$
Z_{n}^{1+}=a Z_{n-1}^{1+}+Z_{n-2}^{1+}-\Sigma_{n-1}
$$

where we have defined

$$
\Sigma_{n-1}=\sum_{j=0}^{a-1} \sigma_{n-1}^{j}
$$

This is a second-order inhomogeneous recurrence relation with solution

$$
Z_{n}^{1+}=G_{n} Z_{1}^{1+}+G_{n-1} Z_{0}^{1+}-\sum_{i=2}^{n} G_{n+1-i} \Sigma_{i-1} .
$$


If we now require $Z_{n}$ to be a periodic orbit of period $p$ of (2.2) then in particular we require $Z_{n}^{1+}$ to have period $p$. The inhomogeneity is merely a sum of discontinuities of $Z_{n}$ and therefore this too has period $p$. Thus imposing periodicity of period $p$ we can arrive at two simultaneous linear equations for $Z_{0}^{1+}$ and $Z_{1}^{1+}$, namely

$$
\begin{aligned}
& Z_{0}^{1+}=Z_{p}^{1+}=G_{p} Z_{1}^{1+}+G_{p-1} Z_{0}^{1+}-\sum_{i=2}^{p} G_{p+1-i} \Sigma_{i-1} \\
& Z_{1}^{1+}=Z_{p+1}^{1+}=G_{p+1} Z_{1}^{1+}+G_{p} Z_{0}^{1+}-\sum_{i=2}^{p+1} G_{p+2-i} \Sigma_{i-1} .
\end{aligned}
$$

When written in matrix form, the determinant the matrix of coefficients of this system is $1+(-1)^{p}-$ $G_{p+1}-G_{p-1} \neq 0$, where we have used the identity $G_{p+1} G_{p-1}-G_{p}^{2}=(-1)^{p}$, readily proved from (1.10). Thus these equations always possess a unique solution. The conclusion is that we may arbitrarily select the discontinuities over a set of periodic orbits of $F$ on the fundamental interval (i.e., select the first columns of the discontinuity matrices), and then define $Z_{0}^{1+}, Z_{1}^{1+}$, i.e., $Z_{0}(1), Z_{1}(1)$, by solving this system of linear equations, and the resulting orbit is periodic on the fundamental interval. By the results of Subsection 2.2 this local data determines the asymptotic (right continuous at 1) globally periodic orbit.

In summary we have the following

Theorem 1. A necessary and sufficient condition for a piecewise-constant, right-continuous at 1 , orbit of (2.2) to be periodic (on the whole of $\mathbb{R}$ ) with period $p$ is that its discontinuities have period $p$ and that $Z_{0}^{1+}, Z_{1}^{1+}$ satisfy equations (2.32) and (2.33).

\section{Discontinuities on $\mathbb{R}$}

Thus far we have a necessary and sufficient condition for orbits of $(2.2)$ to be periodic on the whole of $\mathbb{R}$. We further wish to address the problem of the spatial boundedness of such orbits. To find conditions for spatial boundedness we must understand both the locations and the sizes of the discontinuities of $Z_{n}$ on the whole of $\mathbb{R}$, and that is the purpose of this section. The sizes of the discontinuities will be straightforward to calculate, but identifying their locations presents some difficulty. Since recurrence (2.2) is linear, we may consider the contribution of each periodic orbit, and, indeed, each discontinuity of each periodic orbit, to the global discontinuity set of $Z_{n}$ separately.

We shall return to the issue of spatial boundedness in Section 4. A precise identification of the locations of the discontinuities of $Z_{n}$ will also enable us to calculate the autocorrelation functions. This we shall do in Section 5 .

Let $L_{n}$ denote the set of locations of the discontinuities of $Z_{n}$, i.e.,

$$
L_{n}=\left\{x \in \mathbb{R}: D_{n}(x) \neq 0\right\} .
$$

From $(2.2)$ it is clear that

$$
L_{n}=\bigcup_{j=0}^{a-1} \phi_{1, j}^{-1}\left(L_{n-1}\right) \cup \phi_{2, a}^{-1}\left(L_{n-2}\right),
$$

unless there is a cancellation of discontinuities. As we saw in Subsection 2.2, the global discontinuities of $Z_{n}$ are generated from those in the fundamental interval $I$, and the latter consist of elements of periodic orbits of the map $F$. It is clear from (2.6) that such periodic orbits must be composed of elements of the field

$$
\mathbb{Q}(\omega)=\{x+y \omega: x, y \in \mathbb{Q}\} .
$$


As a consequence, the sets $L_{n}$ consist of elements of $\mathbb{Q}(\omega)$, since the maps $\phi_{1, j}^{-1}, \phi_{2, a}^{-1}$ act on $\mathbb{Q}(\omega)$ as

$$
\begin{aligned}
& \phi_{1, j}^{-1}(x+y \omega)=-(a x+y+a j)-(x+j) \omega, \\
& \phi_{2, a}^{-1}(x+y \omega)=\left(1+a^{2}\right) x+a y-a^{2}+(a x+y-a) \omega .
\end{aligned}
$$

Rather than consider a periodic orbit itself, we shall consider initially an orbit asymptotic to it generated from discontinuity data on the fundamental interval only. By the results of Subsection 2.2 this orbit is eventually periodic and identical to the desired periodic orbit on any bounded subset of $\mathbb{R}$.

For $x \in \mathbb{R}$, we let $\lceil x\rceil$ denote the ceiling of $x$, namely $\min \{n \in \mathbb{Z}: n \geq x\}$, and $\lfloor x\rfloor$ denote the floor of $x$, namely $\max \{n \in \mathbb{Z}: n \leq x\}$. Firstly, we have the following proposition whose proof is virtually identical to the corresponding result in [13].

Proposition 6. The discontinuity location sets $\left(L_{n}\right)$ arising from applying recurrence (2.2) to initial conditions in which $Z_{0}$ has a single discontinuity of size $X$ at $x+y \omega \in I$, and $Z_{1}$ has a discontinuity of size $-X$ at each of $\phi_{1, j}^{-1}(x+y \omega), j=0, \ldots, a-1$, are $L_{0}=\{x+y \omega\}$ and for $n \geq 1$

$$
L_{n}=c^{(n)}+d^{(n)} \omega+M_{n},
$$

where

$$
M_{n}=\left\{\lceil i / \omega\rceil+i \omega: i=l_{n}, \ldots, r_{n}\right\},
$$

with

$$
l_{n}, r_{n}=\left\{\begin{array}{l}
-\left(G_{n+1}-G_{n}\right), G_{n}-1, \quad n \text { odd } ; \\
-G_{n}, G_{n+1}-G_{n}-1, \quad n \text { even },
\end{array}\right.
$$

and where

$$
c^{(n)}=(-1)^{n}\left(G_{n+1} x+G_{n} y\right), \quad d^{(n)}=(-1)^{n}\left(G_{n} x+G_{n-1} y\right) .
$$

Moreover, each discontinuity of $L_{n}$ is of size $(-1)^{n} X$.

We must also consider the case in which $Z_{0}$ has no discontinuities and $Z_{1}$ has a single discontinuity in $I$. However, this situation is clearly identical to that of Proposition 6, but with a reduction of one in all subscripts. More precisely we have

Proposition 7. The discontinuity location sets $\left(L_{n}\right)$ arising from applying recurrence (2.2) to initial conditions in which $Z_{0}$ has no discontinuities and $Z_{1}$ has a single discontinuity of size $-X$ at $x+y \omega \in I$ are $L_{0}=\emptyset, L_{1}=\{x+y \omega\}$, and for $n \geq 2$

$$
L_{n}=c^{(n-1)}+d^{(n-1)} \omega+M_{n-1},
$$

with $c^{(n)}, d^{(n)}, M_{n}$ as in the statement of Proposition 6. Moreover, each discontinuity of $L_{n}$ is of size $(-1)^{n} X$.

Thus far we have considered functions possessing only a finite number of discontinuities. A globally periodic orbit however possesses an infinite number of discontinuities, being the limit (defined by pointwise convergence) of the sets $\left(L_{n}\right)$ considered thus far. It is clear from Proposition 6 that the discontinuity set generated by a single discontinuity at the fixed point zero is

$$
\{\lceil i / \omega\rceil+i \omega: i \in \mathbb{Z}\},
$$

this being the limit of the corresponding sets $L_{n}$ with $a=b=0$, so that $c^{(n)}=d^{(n)}=0$ for all $n$. However care is needed in the determination of these sets for other periodic orbits. We shall return to this issue in Section 5 where we consider the periodic orbit of (2.2) generated by the periodic orbit of $F$ containing $1 / 2$. 


\section{Global boundedness}

From Propositions 6 and 7 we observe that each discontinuity generates an ordered set of discontinuities of the same size and sign in which the elements of each set have identical relative displacements: the elements of each set are separated from each other by the same amount $(a+\omega$ or $1+a+\omega)$ in the same order. Moreover, with the exception of the fixed point 1 , each nonzero discontinuity causes an unbounded monotonic growth as we move further from the fundamental interval on both sides.

A discontinuity at 1 is different. From Proposition 6 we see that if $Z_{0}$ has a discontinuity at 1 of size $X$ then this discontinuity gives rise to discontinuities in $Z_{n}$ of size $X$ at locations $\geq 1$ for even $n$ and of size $-X$ at locations $\leq-(a+\omega)$ for odd $n$. Proposition 7 shows that if $Z_{1}$ has a discontinuity at 1 of size $-X^{\prime}$ then this discontinuity gives rise to discontinuities in $Z_{n}$ of size $X^{\prime}$ at locations $\leq-(a+\omega)$ for even $n$ and of size $-X^{\prime}$ at locations $\geq 1$ for odd $n$.

For global boundedness we require there to be cumulative growth on neither the left- nor the right-hand sides of the fundamental interval, and as in [13] we are led to the following result.

Theorem 2. A necessary and sufficient criterion for a piecewise-constant periodic orbit of (2.2) to be globally bounded is

$$
\sum_{y \in(-\omega-a+1,1]} D_{1}(y)=\sum_{y \in(1-\omega, 1)} D_{0}(y) \quad \text { and } \quad \sum_{y \in(-\omega-a+1,1)} D_{1}(y)=\sum_{y \in(1-\omega, 1]} D_{0}(y) .
$$

Note that in general the inclusion or exclusion of 1 from the intervals in these sums is crucial. (However, the points $-\omega-a+1$ and $1-\omega$ are never discontinuities.) Further note that when 1 is not a discontinuity location these two conditions are identical.

From the physical point of view we deduce that we may choose many forms for the modulation function, and, provided the conditions for periodicity (Theorem 1) and global boundedness (Theorem 2) are satisfied, the resulting autocorrelation function will display asymptotic self-similarity. More precisely, provided the function $\Phi$ is chosen so that the initial conditions $Z_{0}(x)=0, Z_{1}(x)=\Phi(-\omega x)$ are periodic or preperiodic on the fundamental interval $I$, and that two successive functions on this periodic orbit satisfy the periodicity and global boundedness conditions, then there is asymptotic self-similarity of the autocorrelation function.

\section{Example}

As a further application of our work in Section 3, we now look in detail at the the particular choice of modulation function (1.21) briefly mentioned in the introduction. When $a$ is even, a period-2 orbit results as shown in Figure 1 in the case $a=2$. A period- 6 arises when $a$ is odd. In the case $a=1$ the discontinuities of the initial conditions (1.19) do not all form discontinuities of the period-6 orbit. In particular, the function $Z_{1}(x)=\Phi(-\omega x)$ has a discontinuity at $x=\omega^{-1} / 2 \in I$ which is not periodic for $F$, but its image under $F$ is $1 / 2$ which is part of the period-two orbit $\{1 / 2,-\omega / 2\}$. The case $a=1$ having been treated in [13], we shall only consider the cases $a>1$. In contrast to the case $a=1$ we have $\omega^{-1} / 2 \notin I$ and $-\omega^{-1} / 2=-(a+\omega) / 2 \in I$, with this latter point being periodic for $F$. In all cases $Z_{1}$ also has a discontinuity in the fundamental interval at the fixed point 0 .

Henceforth, when we refer to the orbit $\left(Z_{n}\right)$ we mean the global periodic orbit, and not the orbit asymptotic to it that we generate from the periodic orbit on the fundamental interval. This global periodic orbit is generated from the restricted discontinuity set equal to the union of Examples 1 and 2. When $a$ is even this is $\{1 / 2,-(a+\omega) / 2\} \cup\{0\}$ with discontinuity values $X_{0}^{0}=-2, X_{1}^{0}=0$, corresponding to $\{1 / 2,-(a+\omega) / 2\}$, 
and $X_{0}^{1}=2$ corresponding to $\{0\}$. This data means that $Z_{0}$ has discontinuities $X_{0}^{0}=-2$ at $1 / 2,-X_{1}^{0}=0$ at $-(a+\omega) / 2$, and $X_{0}^{1}=2$ at 0 , whilst $Z_{1}$ has discontinuities $X_{1}^{0}=0$ at $1 / 2,-X_{0}^{0}=+2$ at $-(a+\omega) / 2$, and $-X_{0}^{1}=-2$ at 0 . The point 1 is not a discontinuity point, and to guarantee periodicity we specify $Z_{0}^{1+}=$ $Z_{0}(1)=0$, and $Z_{1}^{1+}=Z_{1}(1)=-1$. We further note that this data satisfies the condition $X_{1}^{0}-X_{0}^{0}-X_{0}^{1}=0$, which is the criterion of the previous section (Theorem 2) guaranteeing a globally bounded periodic orbit.

When $a$ is odd greater than 1 the restricted discontinuity set is $\{1 / 2,-(a+\omega) / 2,-(a-1+\omega) / 2\} \cup\{0\}$ with discontinuity values $X_{0}^{0}=-2, X_{1}^{0}=0, X_{2}^{0}=0$, corresponding to $\{1 / 2,-(a+\omega) / 2,-(a-1+\omega) / 2\}$, and $X_{0}^{1}=2$ corresponding to $\{0\}$. This data means that $Z_{0}$ has discontinuities $X_{0}^{0}=-2$ at $1 / 2, X_{2}^{0}=0$ at $-(a+\omega) / 2,-X_{1}^{0}=0$ at $-(a-1+\omega) / 2$, and $X_{0}^{1}=2$ at 0 , whilst $Z_{1}$ has discontinuities $X_{1}^{0}=0$ at $1 / 2,-X_{0}^{0}=+2$ at $-(a+\omega) / 2,-X_{2}^{0}=0$ at $-(a+\omega) / 2$, and $-X_{0}^{1}=-2$ at 0 . Again, the point 1 is not a discontinuity point, and to guarantee periodicity we specify $Z_{0}^{1+}=Z_{0}(1)=0$, and $Z_{1}^{1+}=Z_{1}(1)=-1$. We further note that this data satisfies the condition $X_{1}^{0}-X_{0}^{0}-X_{2}^{0}-X_{0}^{1}=0$, guaranteeing a globally bounded periodic orbit (Theorem 2).

The value of the corresponding autocorrelation function $K_{P_{y}}$ at resonance times $G_{n}$ will be calculated explicitly in terms of the amplitude $\kappa$. In the case $n \equiv 1 \bmod 2$, since (as we shall show) $Z_{n}= \pm 1$, equation (1.20) gives us $K_{P_{y}}\left(G_{n}\right)=\cos \kappa(=0$ when $\kappa=\pi / 2)$. In the case $n \equiv 0$ mod 2 where now (again, as we shall show) $Z_{n}=0, \pm 2$ we have $K_{P_{y}}\left(G_{n}\right)=1-\alpha+\alpha \cos 2 \kappa(=1-2 \alpha$ when $\kappa=\pi / 2)$ where (as we shall establish) $2 \alpha=1 / \sqrt{a^{2}+4}$ is the asymptotic average value of $\left|Z_{n}\right|$. Figure 2 shows $K_{P_{y}}$ when $a=2$ for the case $\kappa=\pi / 2$. As a consequence of our results when $n \equiv 0 \bmod 2$ we have $K_{P_{y}}\left(G_{n}\right) \rightarrow 1-1 / \sqrt{8}=$ $0.646446 \ldots$ as $n \rightarrow \infty$.

\subsection{The discontinuity location sets}

From Proposition 6 it is immediate that the discontinuity location set associated with the fixed point 0 is

$$
L^{1}=\{\lceil i / \omega\rceil+i \omega: i \in \mathbb{Z}\}
$$

When $a$ is odd (greater than 1) $1 / 2$ is part of the period-three orbit $\{1 / 2,-(a+\omega) / 2,-(a-1+\omega) / 2\}$. When $a$ is even it is part of the period-two orbit $\{1 / 2,-(a+\omega) / 2\}$. The golden mean case $a=1$ is slightly different, and was dealt with in detail in [13].

We define the following sets:

$$
\begin{aligned}
& L_{\text {odd }}^{0,0}=\{\lceil i / \omega+1 / 2\rceil-1 / 2+i \omega: i \in \mathbb{Z}\}, \\
& L_{\text {odd }}^{0,1}=\{\lceil(i-1 / 2) / \omega+1 / 2\rceil-1 / 2+(i-1 / 2) \omega: i \in \mathbb{Z}\}, \\
& L_{\text {odd }}^{0,2}=\{\lceil(i-1 / 2) / \omega\rceil+(i-1 / 2) \omega: i \in \mathbb{Z}\},
\end{aligned}
$$

and

$$
\begin{aligned}
& L_{\text {even }}^{0,0}=\{\lceil i / \omega+1 / 2\rceil-1 / 2+i \omega: i \in \mathbb{Z}\}, \\
& L_{\text {even }}^{0,1}=\{\lceil(i-1 / 2) / \omega\rceil+(i-1 / 2) \omega: i \in \mathbb{Z}\} .
\end{aligned}
$$

Note that $L_{\text {even }}^{0,0}=L_{\text {odd }}^{0,0}, L_{\text {even }}^{0,1}=L_{\text {odd }}^{0,2}$, and that $L_{\text {odd }}^{0,0}, L_{\text {odd }}^{0,1}, L_{\text {odd }}^{0,2}$ are the sets defined in the golden mean analysis of [13].

Definition. We say that the limit of a sequence of sets $L_{n}=\left\{a_{n}(i): i \in I_{n}\right\}$, where $I_{n}$ is a finite sequence of consecutive integers, is equal to a set $L=\{a(i): i \in \mathbb{Z}\}$, and write $L_{n} \rightarrow L$, whenever there exist integer sequences $\left(l_{n}\right),\left(r_{n}\right)$ such that $L \cap L_{n}=\left\{a(i): i=l_{n}, \ldots, r_{n}\right\}$ with $l_{n} \rightarrow-\infty, r_{n} \rightarrow \infty$ as $n \rightarrow \infty$. 
Then we have the following proposition whose proof is similar to the corresponding proof in [13].

Proposition 8. The discontinuity location sets $L_{n}$ generated by the periodic orbit containing $1 / 2$ satisfy

1. $a$ odd

$$
L_{n} \rightarrow L_{o d d}^{0, k}, \quad n \equiv k \quad \bmod 3 ;
$$

2. a even

$$
L_{n} \rightarrow L_{\text {even }}^{0, k}, \quad n \equiv k \quad \bmod 2 .
$$

\subsection{Combining the discontinuities}

Comparing (5.1) with (5.2-5.4) and (5.5-5.6) we see that the discontinuity location sets generated from the periodic orbit containing $1 / 2$ are disjoint from those associated with the fixed point 0 . Thus, there can be no cancellation, or even modification, of the discontinuities generated by one periodic orbit by the other. We further recall that the elements of each of these sets form an increasing sequence with separation either $a+\omega$ or $1+a+\omega$.

The initial conditions for the discontinuities are $X_{0}^{0}=-2, X_{1}^{0}=0$, and, when present, $X_{2}^{0}=0$, and $X_{0}^{1}=2$. It follows that each discontinuity of $Z_{n}$ due to the periodic orbit containing $1 / 2$ is of size -2 when $n$ is even, and of size +2 when $n$ is odd. Each discontinuity of $Z_{n}$ due to the fixed point 0 is of size +2 when $n$ is even, and of size -2 when $n$ is odd.

As well as specifying the initial discontinuity data, we have also specified the initial values $Z_{0}(1)=0$ and $Z_{1}(1)=-1$, thereby giving us initial conditions asymptotic to a globally bounded periodic orbit of period-2 when $a$ is even and period- 6 when $a$ is odd. In the case of odd $a$, it is straightforward to check that $Z_{2}(1)=1$, $Z_{3}(1)=0, Z_{4}(1)=1, Z_{5}(1)=-1$, so that, knowing from Section 2 that the discontinuities have period-6 satisfying $D_{n+3}=-D_{n}$, the periodic orbit $\left(Z_{n}\right)$ itself satisfies $Z_{n+3}=-Z_{n}$.

\subsection{Calculation of the autocorrelation function}

Given this detailed knowledge of the locations and sizes of all discontinuities we can calculate the autocorrelation function (1.20).

Theorem 3. With modulation function given by (1.21), the autocorrelation function $K_{P_{y}}$ satisfies

1. for a even

$$
\lim _{n \rightarrow \infty} K_{P_{y}}\left(G_{n}\right)=\left\{\begin{array}{lll}
1-\alpha+\alpha \cos 2 \kappa, & n \equiv 0 & \bmod 2 ; \\
\cos \kappa, & n \equiv 1 & \bmod 2 ;
\end{array}\right.
$$

2. for a odd

$$
\lim _{n \rightarrow \infty} K_{P_{y}}\left(G_{n}\right)= \begin{cases}1-\alpha+\alpha \cos 2 \kappa, & n \equiv 0 \quad \bmod 3 ; \\ \cos \kappa, & n \equiv 1,2 \bmod 3 ;\end{cases}
$$

where

$$
\alpha=\frac{1}{2 \sqrt{a^{2}+4}} .
$$

For the special case $\kappa=\pi / 2$ in the case $a=2$ shown in Figure 2 we thus have $K_{P_{y}}\left(G_{n}\right) \rightarrow 1-1 / \sqrt{8}=$ $0.646446 \ldots$ as $n \rightarrow \infty$ with $n \equiv 0 \bmod 2$. 
Proof. We shall give the proof for even $a$ only, the odd case being similar.

We denote the discontinuity locations associated with the fixed point 0 given in (5.1) as $a_{i}^{1}+b_{i}^{1} \omega$, and those associated with the period-2 orbit $\{1 / 2,-(a+\omega) / 2\}$ as $a_{i}^{0}+b_{i}^{0} \omega$.

When $n \equiv 0$ mod 2 we see from (5.1) and (5.5) that discontinuities may be matched by the coefficients of $\omega$. The separation of matched discontinuities is then

$$
\begin{aligned}
a_{i}^{1}+b_{i}^{1} \omega-\left(a_{i}^{0}+b_{i}^{0} \omega\right) & =\lceil i / \omega\rceil+i \omega-(\lceil i / \omega+1 / 2\rceil-1 / 2+i \omega) \\
& =1 / 2+\lceil i / \omega\rceil-\lceil i / \omega+1 / 2\rceil= \pm 1 / 2
\end{aligned}
$$

and these discontinuities occur in strict alternation since the separation of elements in each sequence is either $a+\omega$ or $1+a+\omega$.

Now, since $n$ is even, each discontinuity arising from $\{1 / 2,-(a+\omega) / 2\}$ has size $X_{0}^{0}=-2$, whilst each discontinuity arising from $\{0\}$ has size $X_{0}^{1}=2$. Moreover, we have that $Z_{n}(1)=0$, so that the matched discontinuities combine to give an interval of height \pm 2 and width $1 / 2$ in the graph of $Z_{n}$, as in the left-hand graph in Figure 1. For instance, the first pair after 1 is $a+1 / 2+\omega$ with discontinuity -2 , and $a+1+\omega$ with discontinuity +2 . Thus the graph of $Z_{n}$ consists of intervals at height zero punctuated by excursions of width $1 / 2$ to a height \pm 2 .

Now

$$
\cos \kappa Z_{n}(y)= \begin{cases}1, & Z_{n}(y)=0 \\ \cos 2 \kappa, & \left|Z_{n}(y)\right|=2,\end{cases}
$$

and so, defining

$$
\alpha_{n}=\frac{1}{(-\omega)^{-n}} \int_{0}^{(-\omega)^{-n}} \frac{\left|Z_{n}(y)\right|}{2} d y,
$$

as the proportion of the interval $\left[0, \omega^{-n}\right]$ for which $\left|Z_{n}(y)\right|=2,(1.20)$ becomes

$$
K_{P_{y}}\left(G_{n}\right)=1-\alpha_{n}+\alpha_{n} \cos 2 \kappa .
$$

Now

$$
G_{n}=\frac{\omega^{-n}}{\sqrt{a^{2}+4}}+O(1)
$$

from which it is straightforward to deduce that

$$
\omega^{-n}=G_{n+1}+G_{n} \omega+O(1) .
$$

Now $G_{n+1}+G_{n} \omega$ is a discontinuity point generated by the fixed point 0 , and thus the interval $\left[0, \omega^{-n}\right]$ contains $G_{n}+O(1)$ intervals of length $1 / 2$ with $\left|Z_{n}\right|=2$, and thus

$$
\alpha_{n}=\frac{G_{n}+O(1)}{2 \omega^{-n}} \rightarrow \frac{1}{2 \sqrt{a^{2}+4}} .
$$

Hence the result.

When $n \equiv 1$ mod 2 the discontinuity location sets are given by (5.1) and (5.6). We have

$$
\begin{aligned}
a_{i}^{1}+b_{i}^{1} \omega-\left(a_{i}^{0}+b_{i}^{0} \omega\right) & =\lceil i / \omega\rceil+i \omega-(\lceil(i-1 / 2) / \omega\rceil+(i-1 / 2) \omega) \\
& =\omega / 2+\lceil i / \omega\rceil-\left\lceil i / \omega-\omega^{-1} / 2\right\rceil>0
\end{aligned}
$$

and

$$
\begin{aligned}
a_{i+1}^{0}+b_{i+1}^{0} \omega-\left(a_{i}^{1}+b_{i}^{1} \omega\right) & =\lceil(i+1 / 2) / \omega\rceil+(i+1 / 2) \omega-(\lceil i / \omega\rceil+i \omega) \\
& =\left\lceil i / \omega+\omega^{-1} / 2\right\rceil-\lceil i / \omega\rceil+\omega / 2>0,
\end{aligned}
$$


so that the two sets of discontinuities alternate in a strict manner. (Note now that the distance between discontinuities may be $a / 2+\omega / 2$ or $1+a / 2+\omega / 2$. See the right-hand graph of Figure 1.)

Each discontinuity from $\{1 / 2,-(a+\omega) / 2\}$ has size $-X_{0}^{0}=2$, whilst each discontinuity from $\{0\}$ has size $-X_{0}^{1}=-2$. We further have $Z_{n}(1)=-1$ in this case, and the first discontinuity after 1 is $1+a / 2+\omega / 2$ at which $Z_{n}$ increases to +1 . Thus $Z_{n}$ oscillates between values \pm 1 with discontinuity locations as calculated above. It is then a simple consequence of the fact that $Z_{n}= \pm 1$ that the autocorrelation function value $K_{P_{y}}\left(G_{n}\right)$ in equation (1.20) equals $\cos \kappa$.

\section{Conclusion}

In this article we have developed an analysis of the self-similarity properties of the autocorrelation function for a two-level quantum system that is quasiperiodically forced with quadratic irrational frequency $\omega=$ $\left(\sqrt{a^{2}+4}-a\right) / 2$ with piecewise-constant discontinuous modulation function. This work is a generalization of our earlier work in [13], in which the golden-mean case $(a=1)$ was studied.

As with studies of the self-similar fluctuations of the localized eigenstates of the Harper equation [7], the birth of a strange nonchaotic attractor [9], the correlation function of billiards with a partial barrier [16], and the autocorrelation function of a strange nonchaotic attractor [4], this self-similarity may be understood in terms of a functional recurrence, the dynamics of which are driven by a chaotic piecewise-linear map of an interval $[1,11,12,14]$.

Our work here has involved a detailed study of the piecewise-constant periodic orbits of the recurrence (1.1) through the evolution of their discontinuity locations under the recurrence. For periodic behaviour, the discontinuities must comprise a finite set of periodic orbits of the map $F$, the chaotic dynamics of which may be understood in terms of the symbolic dynamics of codes under the shift map. For the special case of modulation function given by (1.21), the limiting behaviour of the principal peaks of the autocorrelation function has been calculated explicitly and the resulting periodic orbit has been fully analysed. We have also determined precise criteria for global spatial boundedness of the periodic orbits.

Quadratic irrationals of the type considered in this article have period-1 continued-fraction expansions, and it is expected that our analysis will extend readily to all (eventually) periodic continued fractions, and thus to all quadratic irrational frequencies $\omega$. A harder problem is the extension of this work to more general irrational frequencies. For non-periodic infinite continued fractions, self-similarity is not to be expected; rather the recurrence (1.1) and the autocorrelation function will most likely display a spatial-temporal chaos, corresponding to a skew-product of the Gauss continued-fraction map on the frequencies $\omega$ and piecewise linear maps $F$ of the form (2.3) with $a$ varying according to the evolution of the Gauss map. Such models will also most likely govern the behaviour for general irrational frequency of other systems considered recently such as $[1,12,14]$, but their analysis presents a considerable challenge for future work in this area.

\section{References}

[1] J. R. Chapman and A. H. Osbaldestin, Self-similar correlations in a barrier billiard, Physica D 180 (2003), 71-91.

[2] A. Crisanti, M. Falcioni, G. Paladin, M. Serva, and A. Vulpiani, Complexity in quantum systems, Phys. Rev. E 50 (1994), 138-144. 
[3] J. Dalton and B. D. Mestel, Renormalization for the Harper equation for quadratic irrationals, J. Math. Phys. 44 (2003), 4776-4783.

[4] U. Feudel, A. Pikovsky, and A. Politi, Renormalization of correlations and spectra of a strange nonchaotic attractor, J. Phys. A 29 (1996), 5297-5311.

[5] U. Feudel, A.S. Pikovsky, and M. A. Zaks, Correlation properties of a quasiperiodically forced two-level system, Phys. Rev. E 51 (1995), 1762-1769.

[6] C. Grebogi, E. Ott, S. Pelikan, and J. A. Yorke, Strange attractors that are not chaotic, Physica D 13 (1984), 261-268.

[7] J. A. Ketoja and I. I. Satija, Self-similarity and localization, Phys. Rev. Lett. 75 (1995), 2762-2765.

[8] J. A. Ketoja and I. I. Satija, Harper equation, the dissipative standard map and strange nonchaotic attractors: Relationship between an eigenvalue problem and iterated maps, Physica D 109 (1997), 7080 .

[9] S. P. Kuznetsov, A. S. Pikovsky, and U. Feudel, Birth of a strange nonchaotic attractor: A renormalization group analysis, Phys. Rev. E 51 (1995), R1629-R1632.

[10] J. M. Luck, H. Orland, and U. Smilansky, On the response of a two-level quantum system to a class of time-dependent quasiperiodic perturbations, J. Stat. Phys. 53 (1988), 551-564.

[11] B. D. Mestel, A. H. Osbaldestin, and B. Winn, Golden mean renormalisation for the Harper equation: the strong coupling fixed point, J. Math. Phys. 41 (2000), 8304-8330.

[12] B. D. Mestel and A. H. Osbaldestin, Periodic orbits of renormalisation for the correlations of strange nonchaotic attractors, Math. Phys. EJ 6 (2000), no. 5, 27 pp.

[13] B. D. Mestel and A. H. Osbaldestin, Renormalization analysis of correlation properties in a quasiperiodically forced two-level system, J. Math. Phys. 43 (2002), 3458-3483.

[14] B. D. Mestel and A. H. Osbaldestin, Golden mean renormalization for a generalized Harper equation: the Ketoja-Satija orchid, preprint (2003).

[15] A. S. Pikovsky and U. Feudel, Correlations and spectra of strange non-chaotic attractors, J. Phys. A 27 (1994), 5209-5219.

[16] J. Wiersig, Singular continuous spectra in a pseudointegrable billiard, Phys. Rev. E 62 (2000), R21-R24. 